\title{
Analyses of Intermediate-Stage Hepatocellular Carcinoma Patients Receiving Transarterial Chemoembolization prior to Designing Clinical Trials
}

\author{
Keisuke Koroki $^{a}$ Sadahisa Ogasawara ${ }^{a}$ b Yoshihiko Ooka ${ }^{a}$

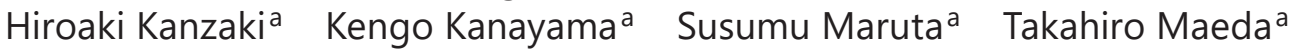 \\ Masayuki Yokoyama ${ }^{a}$ Toru Wakamatsu $^{a}$ Masanori Inoue ${ }^{a}$ \\ Kazufumi Kobayashi ${ }^{a}$ b Soichiro Kiyono $^{a}$ Masato Nakamura ${ }^{a}$ \\ Naoya Kanogawa ${ }^{a}$ Tomoko Saito ${ }^{a}$ Takayuki Kondo ${ }^{a}$ Eiichiro Suzuki ${ }^{a}$ \\ Shingo Nakamoto ${ }^{a}$ Shin Yasui ${ }^{a}$ Akinobu Tawada ${ }^{a, c}$ Tetsuhiro Chiba ${ }^{a}$ \\ Makoto Arai ${ }^{a, c}$ Tatsuo Kanda ${ }^{a}$ d Hitoshi Maruyama ${ }^{\mathrm{e}}$ Jun Kato ${ }^{\mathrm{a}}$ \\ Satoshi Kubokif Masayuki Ohtsukaf Masaru Miyazakif,g \\ Osamu Yokosuka ${ }^{a, h}$ Naoya Kato ${ }^{a, b}$ \\ a Department of Gastroenterology, Graduate School of Medicine, Chiba University, Chiba, \\ Japan; ${ }^{b}$ Translational Research and Development Center, Chiba University Hospital, \\ Chiba, Japan; 'Department of Medical Oncology, Graduate School of Medicine, Chiba

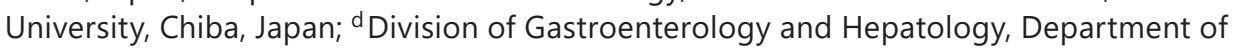 \\ Internal Medicine, Nihon University School of Medicine, Tokyo, Japan; ${ }^{\text {e Department of }}$ \\ Gastroenterology, Juntendo University School of Medicine, Tokyo, Japan; ${ }^{\mathrm{f}}$ Department of \\ General Surgery, Graduate School of Medicine, Chiba University, Chiba, Japan; 9 Digestive \\ Diseases Center, International University of Health and Welfare, Mita Hospital, Tokyo, \\ Japan; ' Department of Internal Medicine, JCHO Funabashi Central Hospital, Funabashi, \\ Japan
}

\section{Keywords}

Hepatocellular carcinoma - Intermediate stage $\cdot$ Transarterial chemoembolization · Durable response rate

\begin{abstract}
Background: Intermediate-stage hepatocellular carcinoma (HCC) has a high frequency of recurrence and progression to advanced stage after transarterial chemoembolization (TACE), particularly in patients with high tumor burden. Promising new results from immune checkpoint inhibitors (ICls) and ICl-based therapies are expected to replace TACE, especially in HCC patients with high tumor burden. Aims: The present study aimed to evaluate the effectiveness
\end{abstract}

K. Koroki, S. Ogasawara, and Y. Ooka contributed equally to this work. 
of TACE with a view to design clinical trials comparing TACE and ICls. Methods: We retrospectively identified intermediate-stage HCC patients undergoing TACE from our database and subdivided patients into low- and high-burden groups based on three subclassification models using the diameter of the maximum tumor and the number of tumors. Clinical outcomes were compared between low- and high-burden intermediate-stage HCC. Results: Of 1,161 newly diagnosed HCC patients, 316 were diagnosed with intermediate-stage disease and underwent TACE. The median overall survival from high-burden intermediate-stage disease was not significantly different by clinical course, reaching high tumor burden in all subclassification models. The prognosis of high-burden patients after initial TACE was poor compared with low-burden patients for two models (except for the up-to-seven criteria). In all three models, high-burden patients showed a poor durable response rate (DRR) both $\geq 3$ months and $\geq 6$ months and poor prognosis after TACE. Moreover, patients with confirmed durable response $\geq 3$ months and $\geq 6$ months showed better survival outcomes for high-burden intermediatestage HCC. Conclusions: Our results demonstrate the basis for selecting a population that would not benefit from TACE and setting DRR $\geq 3$ months or $\geq 6$ months as alternative endpoints when designing clinical trials comparing TACE and ICls.

(C) 2020 The Author(s)

Published by S. Karger AG, Basel

\section{Introduction}

Hepatocellular carcinoma (HCC) is the sixth most common cancer worldwide and the third leading cause of cancer death [1]. For the past several decades, transarterial chemoembolization (TACE) has been one of the most common treatments used for HCC [2]. In 2003, a meta-analysis of six randomized controlled trials demonstrated improved patient survival with TACE compared with best supportive care or suboptimal treatments [3]. Therefore, TACE has become the recommended therapy in patients with intermediate-stage HCC in both Eastern and Western guidelines [4, 5]. Previous reports have indicated that $>50 \%$ of HCC patients who underwent TACE achieved an objective response, as reflected by tumor necrosis; moreover, this objective response was associated with a longer survival duration $[1,6]$. Llovet et al. [7] reported that patients who had achieved and sustained an objective response for at least 6 months had a better prognosis than others. However, TACE is not a curative treatment and may cause liver function deterioration, incomplete tumor necrosis, and the potential risk of extrahepatic metastasis [8]. Although a lot of previous reports indicated the effectiveness of TACE particularly in patients with a small number or small size of intrahepatic lesions (i.e., low-tumor-burden patients), patients with high tumor burden have a high frequency of recurrence and progression to advanced-stage HCC.

In 2007, sorafenib was shown to prolong overall survival (OS) in a phase III clinical trial in advanced HCC in a Western population [9]. This result was replicable in other phase III trials, including advanced HCC patients from Asia-Pacific regions [10]. Strategies to switch from TACE to sorafenib have been advocated in several guidelines [5, 11]. Studies have demonstrated the effectiveness of the conversion from TACE to sorafenib at the time of TACE refractoriness in patients with intermediate-stage HCC $[12,13]$. Moreover, these results were confirmed in a large cohort prospective observation study [14]. Recently, several anticancer agents have become systemic therapies in HCC patients [15-18], and conversion from TACE to systemic therapies has become a more common treatment choice $[5,19]$.

Novel promising results using immune checkpoint inhibitors (ICIs) or ICI-based therapies have been shown in various cancer types [20,21]. In HCC as well as other malignancies, two early-phase studies using ICIs (nivolumab and pembrolizumab) demonstrated that $17-20 \%$ of patients achieved objective response and most confirmed durable response [22,

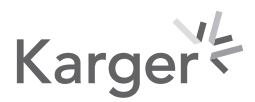


23]. On the basis of these results, both nivolumab and pembrolizumab have already been approved by the Food and Drug Administration in the United States for second-line systemic therapy after sorafenib. Several clinical trials that are using a combination setting with ICIs or ICI-based therapies are currently underway and are expected to show a higher response rate with longer durability in patients with HCC [24]. In 2019, atezolizumab combined with bevacizumab for the first-line treatment of patients with advanced HCC prolonged OS and progression-free survival compared with sorafenib, according to the results of a global phase III trial (IMbrave150) [25]. The result of IMbrave150, the first phase III study of combination setting with ICIs or ICI-based therapies in patients with advanced HCC that released outcomes, showed a large magnitude of impacts that resulted from the combination of atezolizumab and bevacizumab as the first regimen to prove superiority of OS compared with sorafenib, with longer durable responses and tolerable safety profiles. These results suggest that combination setting with ICIs or ICI-based therapies, including atezolizumab combined with bevacizumab, may have the potential to become "a game changer" in the landscape of HCC treatment.

Recently, several articles and guidelines have put forward the need for appropriate selection of patients who can achieve clinical benefit by TACE $[4,5,19,26,27]$. However, there have not been definite or positive results of randomized controlled trials which were comparable to TACE or other promising systemic agents yet. As far as the results of IMbrave150 are concerned, these higher and durable response regimens of ICIs or ICI-based therapies are expected to replace TACE, especially in limited populations of intermediate-stage HCC patients with high tumor burden. Now is the time that clinical trials comparing TACE and ICIbased therapies should be planned. To the best of our knowledge, there are relatively few reports that have become the standard data for TACE that focus on designing clinical trials to compare TACE and ICI-based therapies. The present study aimed to evaluate the effectiveness of TACE in order to design clinical trials and identify limited high-burden populations that could be shifted from TACE to ICI-based therapies.

\section{Patients and Methods}

Patients

We retrospectively retrieved the whole clinical courses of HCC patients who were initially diagnosed at Chiba University Hospital. Intermediate-stage HCC patients ( $>3$ lesions of any size or 2-3 lesions $>30 \mathrm{~mm}$ in diameter without macrovascular invasion or extrahepatic spread and without any cancer-related symptoms) receiving TACE were identified from the cohort. The present study corrected data retrospectively; therefore, ECOG PS could not be confirmed in detail.

\section{Strategy for TACE}

The decision to treat patients using TACE was based on discussions involving multidisciplinary teams at our institution. The teams consisted of experts in both HCC treatments and TACE who had >10 years prior experience. First, we considered whether surgical resection, the most curative treatment, was appropriate [28]. Second, local ablation was considered when technically feasible and when treatment completion was predicted. Thereafter, all the remaining patients were considered for TACE, the first-line noncurative intermediate-stage HCC treatment [12]. All patients received combinations of computed tomography (CT) scans with hepatic arteriography and arterial portography. Tumor-feeding arteries were identified using a threedimensional reconstruction system. TACE was performed using the superselective technique and the procedures were performed on demand. CT scans were performed within 3 months of TACE to evaluate the radiological response of the tumor. Follow-up dynamic enhanced CT or magnetic resonance imaging (MRI) was performed every 3-4 months [29].

If a viable tumor was identified or if there was local or distant intrahepatic recurrence, repeated TACE was considered. If the patients had intrahepatic lesions that were unresponsive to TACE, other treatment options (systemic therapies or hepatic arterial infusion chemotherapy) were considered. If liver function

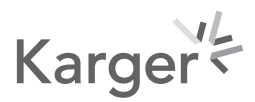




\section{Liver Cancer}

\begin{tabular}{|c|c|}
\hline \multicolumn{2}{|l|}{ Liver Cancer 2020;9:596-612 } \\
\hline DOI: 10.1159/000508809 & $\begin{array}{l}\text { (c) } 2020 \text { The Author(s). Published by S. Karger AG, Basel } \\
\text { www.karger.com/lic }\end{array}$ \\
\hline
\end{tabular}

Koroki et al.: Designing Clinical Trials for Intermediate-Stage HCC

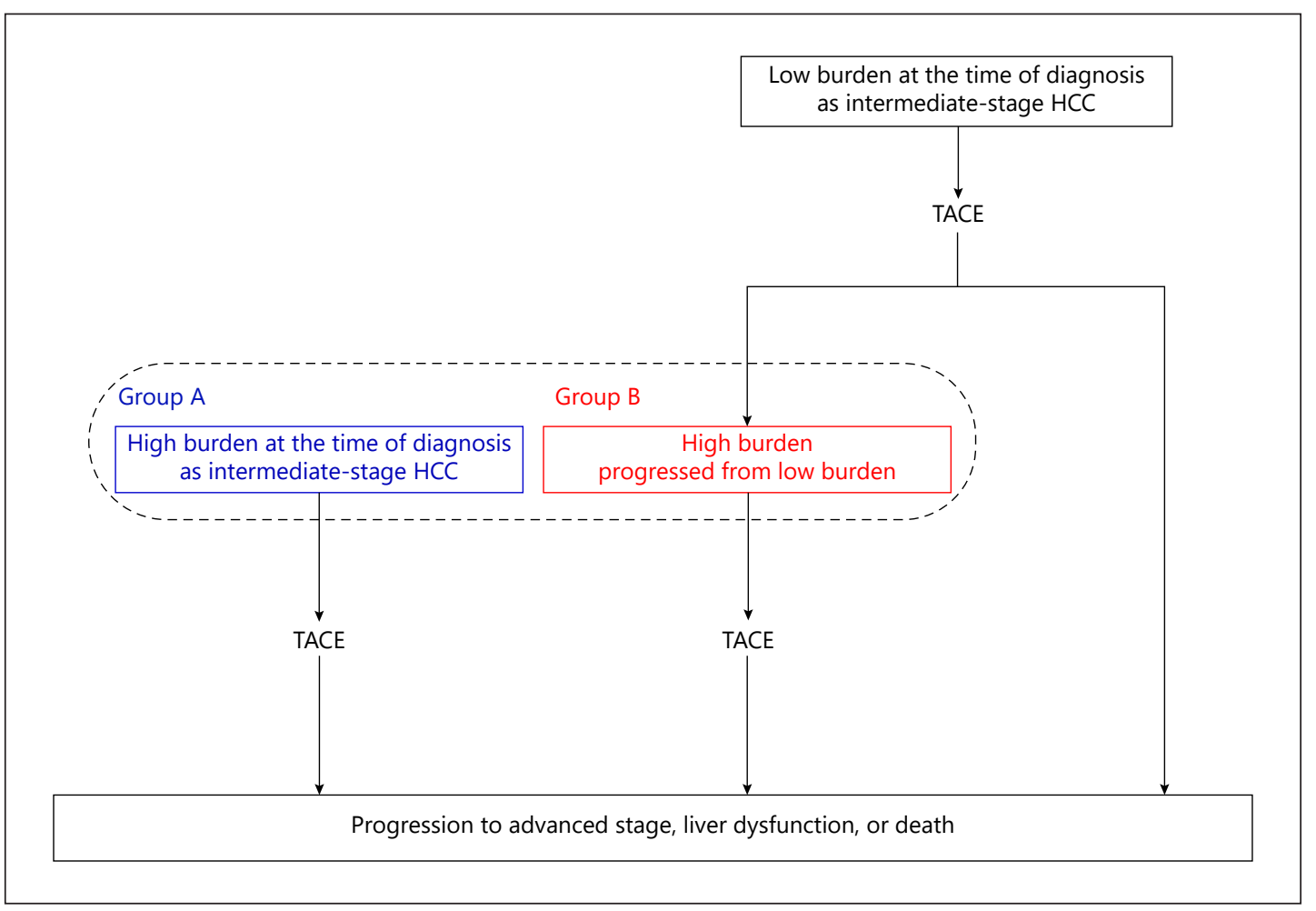

Fig. 1. Subdivision of high-burden intermediate-stage HCC patients receiving TACE into two common clinical courses. Group A: patients with high tumor burden at the time of diagnosis of intermediate-stage disease. Group B: patients with low tumor burden at the time of diagnosis of intermediate-stage disease and progression to high tumor burden during the clinical course. HCC, hepatocellular carcinoma; TACE, transarterial chemoembolization.

deterioration was observed, palliative care was strongly recommended. Repeated TACE was performed for patients with recurrence in whom TACE was expected to be effective.

\section{Subclassification Models for Low and High Tumor Burden}

Low-burden intermediate-stage HCC patients (i.e., low number and small size of lesions) are expected to show better outcomes of TACE compared with high-burden intermediate-stage HCC patients [30, 31]. We previously reported that the number of tumors was independently and significantly associated with the prognosis of intermediate-stage HCC treated with TACE [29]. We divided high-burden intermediate-stage HCC patients receiving TACE into two common clinical courses as follows: (1) patients with high tumor burden at the time of diagnosis of intermediate-stage disease (group A) and (2) patients with low tumor burden at the time of diagnosis of intermediate-stage disease and progression to high tumor burden during the clinical course (group B) (Fig. 1). Three subclassification models of intermediate-stage HCC were defined based on the diameter of the maximum tumor and the number of tumors and verified the clinical outcomes (subclassification model 1, up-to-seven criteria, high burden: largest tumor diameter [cm] + number of tumors > 7; subclassification model 2, five-seven criteria, high burden: 4 lesions $\geq 5 \mathrm{~cm}$ or $>7$ lesions; and subclassification model 3, seven lesions criteria, high burden: >7 lesions) (Table 1).

\section{Radiological Assessments and Durable Response Rate}

We retrospectively evaluated all CT and MRI images after initial TACE. A responder was defined as a patient who achieved a complete or partial response according to the modified Response Evaluation Criteria in Solid Tumors (mRECIST) for HCC [32]. We additionally assessed durable response rate (DRR) $\geq 6$ months (rate of continuous response [complete or partial objective response] beginning within 12 months of treatment and lasting $\geq 6$ months) according to mRECIST $[32,33]$. DRR $\geq 3$ months as well as DRR $\geq 6$ months were evaluated. 
Table 1. Three subclassification models of low/high tumor burden

\begin{tabular}{lll}
\hline Subclassification models & Low burden & High burden \\
\hline Model 1 (up-to-seven criteria) & within up-to-seven ${ }^{1}$ & exceeding up-to-seven $^{2}$ \\
\hline Model 2 (five-seven criteria) & 2 or 3 lesions (any size) & $4-7$ lesions $\geq 5 \mathrm{~cm}$ \\
& $4-7$ lesions $<5 \mathrm{~cm}$ & $>7$ lesions (any size) \\
\hline Model 3 (seven lesions criteria) & $\leq 7$ lesions (any size) & $>7$ lesions (any size) \\
\hline
\end{tabular}

${ }^{1}$ Largest tumor diameter $(\mathrm{cm})+$ number of tumors $\leq 7 .{ }^{2}$ Largest tumor diameter $(\mathrm{cm})+$ number of tumors $>7$.

Table 2. Baseline characteristics of 316 intermediate-stage HCC patients treated with TACE

\begin{tabular}{lc}
\hline Demographics/characteristics & \\
\hline Male sex & $233(73.7 \%)$ \\
Age $>72$ years & $139(44.0 \%)$ \\
Hepatitis C virus infection & $205(64.9 \%)$ \\
Hepatitis B virus infection & $31(9.8 \%)$ \\
Alcohol abuse & $45(14.2 \%)$ \\
Child-Pugh class A & $256(81.0 \%)$ \\
Alpha-fetoprotein $>400 \mathrm{ng} / \mathrm{mL}$ & $52(16.5 \%)$ \\
Number of tumors & \\
$\quad 2-3$ & $90(28.5 \%)$ \\
$\quad 4-7$ & $113(35.8 \%)$ \\
$\quad 7$ & $113(35.8 \%)$ \\
Maximum tumor size $\geq 5 \mathrm{~cm}$ & $68(21.5 \%)$ \\
Initially diagnosed as intermediate-stage & $178(56.3 \%)$ \\
Pretreatment & $138(43.7 \%)$ \\
\hline
\end{tabular}

Values are presented as $n(\%)$. HCC, hepatocellular carcinoma; TACE, transarterial chemoembolization.

\section{Statistical Analysis}

The Pearson $\chi^{2}$ test was used to compare demographic and clinical characteristics. OS was defined as the time TACE was performed until death, and the censoring date was defined as the date of the last followup. Kaplan-Meier plots of medians with $95 \%$ confidence intervals (CIs) were used to estimate OS. A cumulative progression rate to the advanced stage was defined as the date from initial TACE to the date of progression to the advanced stage with either macrovascular invasion or extrahepatic metastasis being identified by radiological assessments. The censoring date was defined as the date of the last radiological assessment. All $p$ values $<0.05$ were considered statistically significant. All statistical analyses were performed using SPSS statistical software version 25 (IBM, Chicago, IL, USA).

\section{Results}

\section{Baseline Characteristics}

Among the 1,161 newly diagnosed HCC patients identified from the database of Chiba University Hospital between 2003 and 2015, we included intermediate-stage HCC patients who had undergone TACE and excluded patients who had received other treatments (resection, hepatic arterial infusion chemotherapy, systemic therapy, and palliative care). Of the 316 patients who underwent TACE for intermediate-stage HCC, 256 were Child-Pugh 

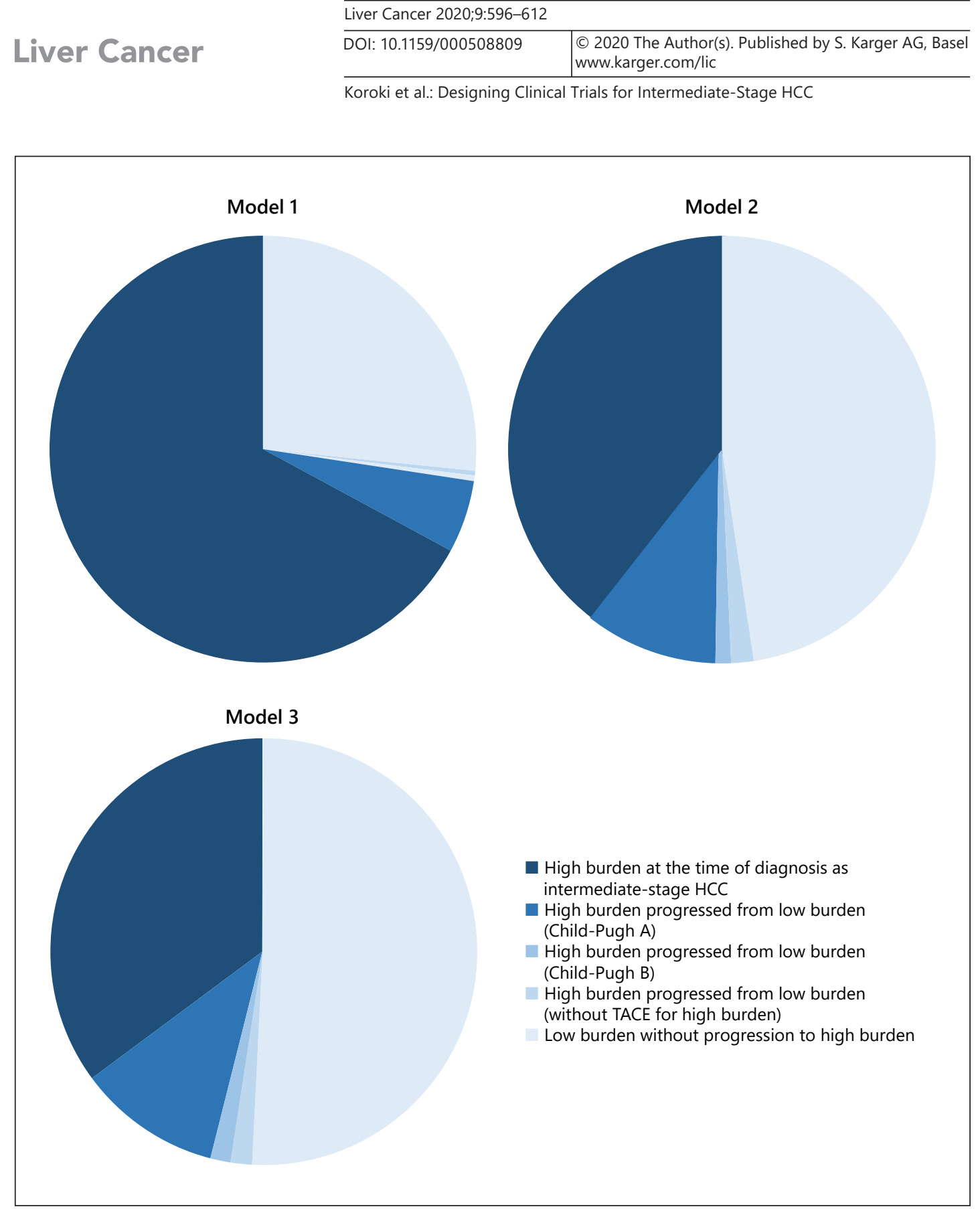

Fig. 2. Patient flow divided by tumor burden in each subclassification model. HCC, hepatocellular carcinoma.

class A, and 138 had previous treatment histories (diagnosed as early-stage HCC and received other treatments prior to progressing to intermediate-stage HCC) (Table 2). The most common etiology was hepatitis $\mathrm{C}$ virus infection (64.9\%), followed by alcohol abuse $(14.2 \%)$ and hepatitis B virus infection (9.8\%). In the present cohort, 52 patients $(16.5 \%)$ had an alpha-fetoprotein (AFP) level $>400 \mathrm{ng} / \mathrm{mL}$. The completion rates of superselective TACE of the whole cohort and Child-Pugh A populations were $91.1 \%$ (288 of 316 patients) and $90.2 \%$ (231 of 256 patients), respectively. The median follow-up period was 20.9 months (95\% CI 18.6-23.2). During the follow-up period, 295 of 316 patient $(93.4 \%)$ and 242 of 256 patients (94.5\%) received two or more radiological assessments within 6 months following TACE. 


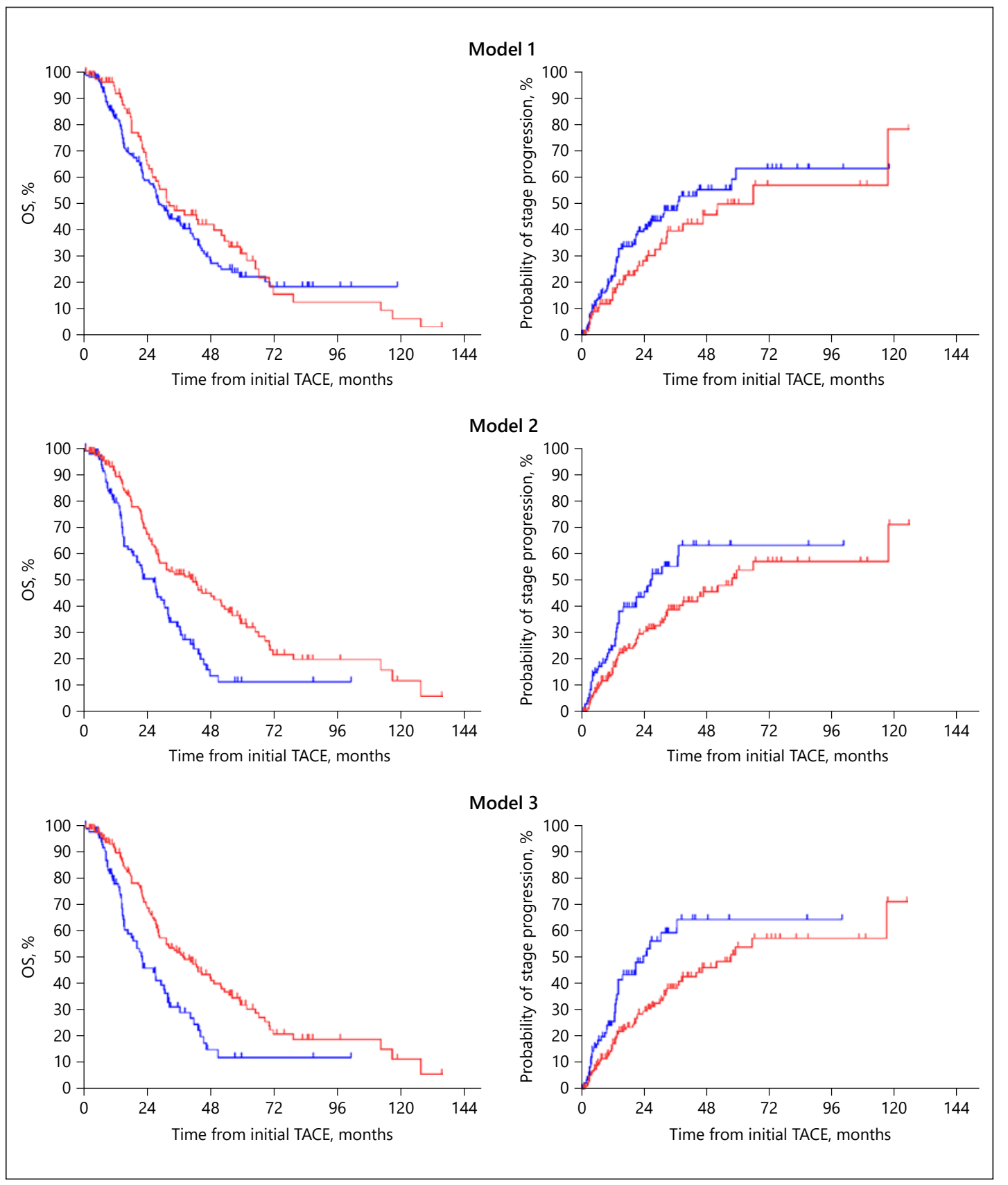

Fig. 3. Kaplan-Meier curves of OS (left) and cumulative progression rates to advanced stages (right) from initial TACE in Child-Pugh A patients with low and high burdens in each subclassification model. Red lines indicate low burden and blue lines high burden. Model 1 (up-to-seven criteria): the median OS of low and high burdens were 32.3 months ( $95 \%$ CI 17.3-47.3) and 28.3 months (95\% CI 24.2-32.4), respectively ( $p=$ 0.316 ); the median cumulative rates to advanced stages of low and high burdens were 52.0 months $(95 \% \mathrm{CI}$ 23.9-80.1) and 37.0 months ( $95 \%$ CI 22.6-51.4), respectively ( $p=0.159$ ). Model 2 (five-seven criteria): the median OS of low and high burdens were 41.3 months (95\% CI 27.8-54.8) and 26.3 months (95\% CI 20.1$32.5)$, respectively $(p<0.001)$; the median cumulative rates to advanced stages of low and high burdens were 57.5 months (95\% CI 38.9-76.1) and 26.4 months (95\% CI 16.0-36.8), respectively ( $p=0.011)$. Model 3 (seven lesions criteria): the median OS of low and high burdens were 38.0 months (95\% CI 28.4-47.6) and 22.1 months ( $95 \%$ CI $14.8-29.4)$, respectively $(p<0.001)$; the median cumulative rates to advanced stages of low and high burdens were 57.5 months (95\% CI 38.9-76.1) and 23.9 months (95\% CI 13.1-34.7), respectively $(p=0.003)$. OS, overall survival; TACE, transarterial chemoembolization. 
Koroki et al.: Designing Clinical Trials for Intermediate-Stage HCC

Comparison of Low and High Tumor Burden and Two Different Clinical Courses of High-Burden Intermediate-Stage HCC Patients Receiving TACE

The median OS in our cohort receiving TACE was 27.2 months (95\% CI 23.3-31.1), 31.1 months (95\% CI 26.1-36.1) in Child-Pugh A patients and 18.6 months (95\% CI 11.9-25.3) in Child-Pugh B patients. Among the 256 Child-Pugh A patients, $67.2 \%$ (172 patients), 39.5\% (101 patients), and 35.2\% (90 patients) were classified as high-burden intermediate-stage HCC at the time of first TACE for subclassification model 1 (up-to-seven criteria), model 2 (five-seven criteria), and model 3 (seven lesions criteria), respectively (Fig. 2). The success rates of superselective TACE in high-burden intermediate-stage HCC patients in subclassification models 1,2 , and 3 were $88.4,84.2$, and $83.3 \%$, respectively ( $p=0.446)$. The prognosis of high-burden intermediate-stage HCC patients from initial TACE in subclassification models 2 and 3 was significantly poorer than that of low-burden intermediate-stage HCC patients (Fig. 3). The cumulative progression rates from first TACE to advanced-stage HCC are shown in Figure 3. As well as OS, there were significant differences between low- and high-burden intermediate-stage HCC patients in subclassification models 2 and 3. The discriminatory abilities in predicting the 1-, 2-, and 3-year survival of each subclassification model were confirmed using ROC curve analysis (online suppl. Table 1; for all online suppl. material, see www. karger.com/doi/10.1159/000508809). Subclassification models 2 and 3 had significantly better prediction values in terms of 3-year survival as compared to model 1 (model 1 vs. 2: $p=0.036$; model 1 vs. $3: p=0.027$ ).

Furthermore, we compared the OS of low- and high-burden disease in Child-Pugh B patients with intermediate-stage HCC as per the three subclassification models. Among the Child-Pugh B patients in each model, the median OS from the time of initial TACE was not significantly different between patients with low- and high-burden disease (online suppl. Fig. 1).

During the clinical course, 16 (6.3\%), 33 (12.9\%), and $36(14.1 \%)$ patients in subclassification models 1,2 , and, 3, respectively, progressed from low- to high-burden intermediatestage HCC after receiving TACE, and 14 (5.5\%), 26 (10.2\%), and $28(10.9 \%)$ patients in subclassification models 1, 2, and 3 maintained liver function as Child-Pugh A the time of progression to high-burden intermediate-stage HCC. Among the Child-Pugh A patients in each model, the median OS from high-burden intermediate-stage disease was not significantly different between patients with high tumor burden at the time of diagnosis of intermediate-stage disease (group A) and patients with low tumor burden at the time of diagnosis of intermediate-stage disease and progression to high tumor burden during the clinical course (group B) (Fig. 4).

Clinical Outcomes of Radiological and AFP Responders with Intermediate-Stage HCC Who Were Receiving TACE (Child-Pugh A)

In the present cohort, 152 of 256 patients (59.4\%) achieved radiological response after TACE (online suppl. Table 2). The frequencies of response in high-burden intermediate-stage HCC according to models 1,2 , and 3 were $57.0,51.5$, and $47.8 \%$, respectively. The OS of responders with high-burden intermediate-stage HCC was significantly longer in all three subclassification models compared to nonresponders.

In the present cohort, the rates of AFP $>400 \mathrm{ng} / \mathrm{mL}$ in high-burden patients of subclassification models 1, 2, and 3 were 14.0, 19.8, and 20.0\%, respectively. Among high-burden intermediate-stage HCC patients whose AFP levels were $\geq 20 \mathrm{ng} / \mathrm{mL}$ in each subclassification model, correlations were assessed between decreasing AFP values ( $>20 \%$ or $>50 \%$ ) and OS (online suppl. Table 3). In the present study, there were no significant differences in OS between absence or presence of decreasing AFP values that were $>20 \%$ and $>50 \%$ in highburden intermediate-stage HCC patients who received TACE in all subclassification models.

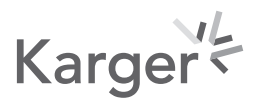




\section{Liver Cancer}

Fig. 4. Kaplan-Meier survival curves from initial TACE in ChildPugh A patients after diagnosis with high-burden disease in each subclassification model. Blue lines indicate high burden at the time of initial diagnosis (group A) and red lines indicate high burden progressing from low burden (group B). Subclassification model 1 (up-to-seven criteria): the median OS of high burden at the time of initial diagnosis and progressing from low burden were 28.3 months (95\% CI 24.2-32.3) and 17.3 months (95\% CI 15.219.4), respectively ( $p=0.129$ ). Model 2 (five-seven criteria): the median OS of high burden at the time of initial diagnosis and progressing from low burden were 26.3 months (95\% CI 20.0-32.5) and 17.3 months (95\% CI 13.0$21.6)$, respectively ( $p=0.510)$. Model 3 (seven lesions criteria): the median OS of high burden at the time of initial diagnosis and progressing from low burden were 22.1 months (95\% CI 14.829.3) and 17.3 months (95\% CI 12.7-21.9), respectively $(p=$ $0.573)$. TACE, transarterial chemoembolization.
Koroki et al.: Designing Clinical Trials for Intermediate-Stage HCC

\section{Model 1}

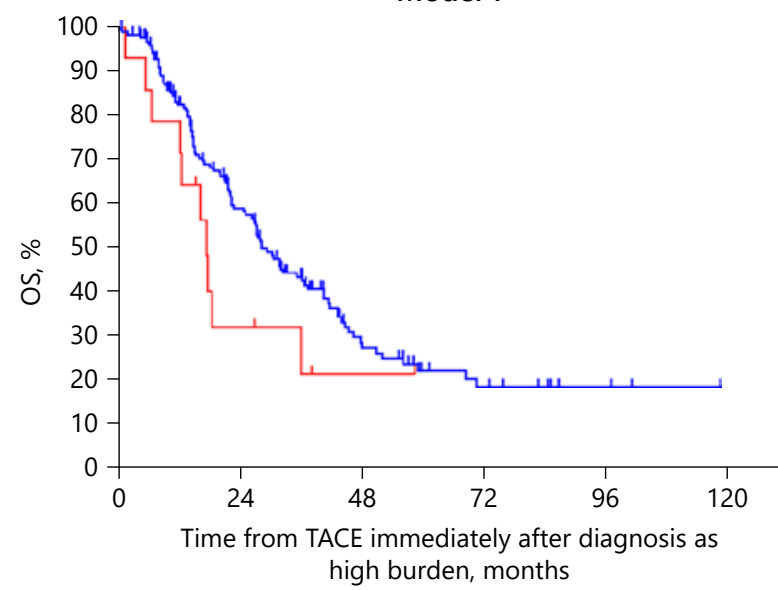

Model 2

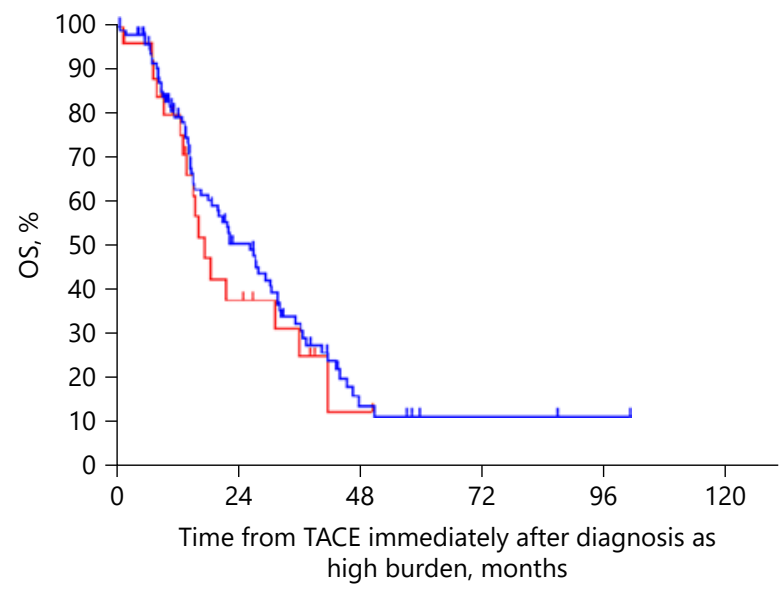

Model 3

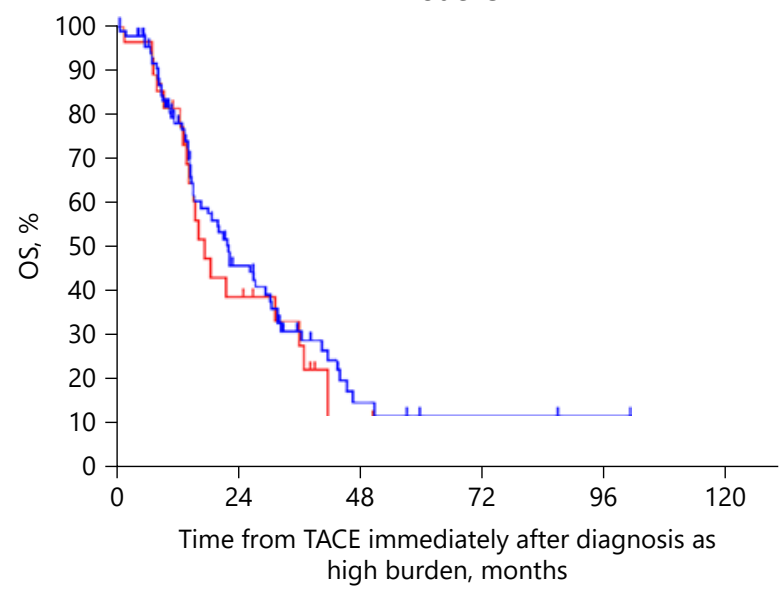


Koroki et al.: Designing Clinical Trials for Intermediate-Stage HCC

Table 3. DRR $\geq 3$ months and $\geq 6$ months for each subclassification model

\begin{tabular}{|c|c|c|c|c|}
\hline & $\begin{array}{l}\text { Low burden (at the time of } \\
\text { diagnosis as intermediate- } \\
\text { stage HCC) }\end{array}$ & $\begin{array}{l}\text { High burden (group A: at } \\
\text { the time of diagnosis as } \\
\text { intermediate-stage HCC) }\end{array}$ & $\begin{array}{l}\text { High burden (group } \\
\text { B: progressed from } \\
\text { low burden) }\end{array}$ & $\begin{array}{l}\text { High burden } \\
\text { (group } A+B \text { ) }\end{array}$ \\
\hline \multicolumn{5}{|c|}{ Model 1 (up-to-seven criteria) } \\
\hline \multicolumn{5}{|l|}{ Child-Pugh A } \\
\hline DRR $\geq 3$ months & $46.4 \%$ & $39.5 \%$ & $28.6 \%$ & $38.7 \%$ \\
\hline DRR $\geq 6$ months & $26.2 \%$ & $15.1 \%$ & $7.1 \%$ & $14.5 \%$ \\
\hline \multicolumn{5}{|l|}{ Child-Pugh B } \\
\hline DRR $\geq 3$ months & $56.3 \%$ & $22.7 \%$ & $0.0 \%$ & $22.2 \%$ \\
\hline DRR $\geq 6$ months & $18.8 \%$ & $9.1 \%$ & $0.0 \%$ & $8.7 \%$ \\
\hline \multicolumn{5}{|c|}{ Model 2 (five-seven criteria) } \\
\hline \multicolumn{5}{|c|}{ Child-Pugh A } \\
\hline DRR $\geq 3$ months & $46.5 \%$ & $34.7 \%$ & $23.1 \%$ & $32.3 \%$ \\
\hline DRR $\geq 6$ months & $24.5 \%$ & $9.9 \%$ & $15.4 \%$ & $11.0 \%$ \\
\hline \multicolumn{5}{|l|}{ Child-Pugh B } \\
\hline DRR $\geq 3$ months & $37.1 \%$ & $24.0 \%$ & $0.0 \%$ & $23.1 \%$ \\
\hline DRR $\geq 6$ months & $14.3 \%$ & $8.0 \%$ & $0.0 \%$ & $6.9 \%$ \\
\hline \multicolumn{5}{|c|}{ Model 3 (seven lesions criteria) } \\
\hline \multicolumn{5}{|l|}{ Child-Pugh A } \\
\hline DRR $\geq 3$ months & $45.8 \%$ & $34.4 \%$ & $21.4 \%$ & $31.4 \%$ \\
\hline DRR $\geq 6$ months & $23.5 \%$ & $10.0 \%$ & $14.3 \%$ & $11.0 \%$ \\
\hline \multicolumn{5}{|l|}{ Child-Pugh B } \\
\hline DRR $\geq 3$ months & $37.8 \%$ & $21.7 \%$ & $0.0 \%$ & $20.8 \%$ \\
\hline $\mathrm{DRR} \geq 6$ months & $16.2 \%$ & $4.3 \%$ & $0.0 \%$ & $3.6 \%$ \\
\hline
\end{tabular}

DRR, durable response rate; HCC, hepatocellular carcinoma.

$D R R \geq 3$ and $\geq 6$ Months in Intermediate-Stage HCC Patients Receiving TACE

Table 3 shows the DRR $\geq 3$ months and $\geq 6$ months of TACE in Child-Pugh A and B patients with low- and high-burden intermediate-stage HCC. In low-burden intermediate-stage HCC patients with Child-Pugh A and B, DRR $\geq 3$ months was 46.4 and $56.3 \%$ for subclassification model $1,46.5$ and $37.1 \%$ for model 2 , and 45.8 and $37.8 \%$ for model 3, respectively. Similarly, DRR $\geq 6$ months was 26.2 and $18.8 \%$ for subclassification model $1,24.5$ and $14.3 \%$ for model 2 , and 23.5 and $16.2 \%$ for model 3. On the other hand, in Child-Pugh A and B patients with high burden at the time of initial diagnosis as intermediate-stage HCC, DRR $\geq 3$ months was 39.5 and $22.7 \%$ for subclassification model $1,34.7$ and $24.0 \%$ for model 2 , and 34.4 and $21.7 \%$ for model 3. Likewise, DRR $\geq 6$ months was 15.1 and $9.1 \%$ for subclassification model $1,9.9$ and $8.0 \%$ for model 2 , and 10.0 and $4.3 \%$ for model 3 . The median OS of TACE in highburden intermediate-stage patients achieving durable response $\geq 3$ months and $\geq 6$ months was mostly significantly longer for all subclassification models (Table 4).

Figure 5 shows the distribution map of tumor burden of the three subclassification models. Among Child-Pugh A patients receiving TACE, limited patients exceeding the up-toseven criteria (category II patients in Fig. 5) showed similar DRR $\geq 6$ months and median OS compared with patients who were not exceeding the up-to-seven criteria (category I in Fig. 5) (category I: OS 32.3 months [95\% CI 17.3-47.3], DRR $\geq 6$ months 26.2\%; category II: OS 44.7 months (95\% CI 32.6-56.8), DRR $\geq 6$ months 22.5\%] (online suppl. Table 4). On the other hand, category IV patients (classified as high-burden intermediate-stage HCC in subclassification model 3) showed a shorter survival period and a lower DRR $\geq 6$ months compared with the other categories (Category IV: OS 22.1 months [95\% CI 14.8-29.4], DDR $\geq 6$ months $10.0 \%)$.

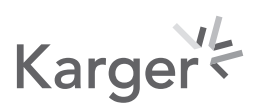




\begin{tabular}{l|l}
\hline Liver Cancer 2020;9:596-612 \\
\hline DOI: 10.1159/000508809 & $\begin{array}{l}\text { @ 2020 The Author(s). Published by S. Karger AG, Basel } \\
\text { www.karger.com/lic }\end{array}$ \\
\hline
\end{tabular}

Table 4. OS in high-burden intermediate-stage HCC patients who achieved durable response $\geq 3$ and $\geq 6$ months (Child-Pugh A)

\begin{tabular}{|c|c|c|c|}
\hline & OS, months & $95 \% \mathrm{CI}$ & $p$ value \\
\hline \multicolumn{4}{|l|}{ Model 1} \\
\hline \multicolumn{4}{|l|}{ Group A } \\
\hline \multicolumn{4}{|c|}{ Durable response $\geq 3$ months } \\
\hline Present & 47.7 & $40.7-54.7$ & $<0.001$ \\
\hline Absent & 21.8 & $15.6-28.0$ & \\
\hline \multicolumn{4}{|c|}{ Durable response $\geq 6$ months } \\
\hline Present & 68.4 & undefined & $<0.001$ \\
\hline Absent & 26.7 & $22.5-31.0$ & \\
\hline \multicolumn{4}{|l|}{ Group $A+B$} \\
\hline \multicolumn{4}{|c|}{ Durable response $\geq 3$ months } \\
\hline Present & 47.7 & $42.6-52.8$ & $<0.001$ \\
\hline Absent & 20.0 & $15.0-25.0$ & \\
\hline \multicolumn{4}{|c|}{ Durable response $\geq 6$ months } \\
\hline Present & 68.5 & undefined & $<0.001$ \\
\hline Absent & 25.0 & $20.7-29.3$ & \\
\hline \multicolumn{4}{|l|}{ Model 2} \\
\hline \multicolumn{4}{|l|}{ Group A } \\
\hline \multicolumn{4}{|c|}{ Durable response $\geq 3$ months } \\
\hline Present & 32.4 & $25.3-39.6$ & 0.014 \\
\hline Absent & 16.5 & $11.4-21.7$ & \\
\hline \multicolumn{4}{|c|}{ Durable response $\geq 6$ months } \\
\hline Present & 41.6 & $19.5-63.6$ & 0.057 \\
\hline Absent & 22.2 & $15.3-29.1$ & \\
\hline \multicolumn{4}{|l|}{ Group $A+B$} \\
\hline \multicolumn{4}{|c|}{ Durable response $\geq 3$ months } \\
\hline Present & 36.3 & $23.5-49.1$ & $<0.001$ \\
\hline Absent & 15.5 & $12.2-18.8$ & \\
\hline \multicolumn{4}{|c|}{ Durable response $\geq 6$ months } \\
\hline Present & 47.7 & $36.8-58.6$ & 0.005 \\
\hline Absent & 21.0 & $16.3-25.6$ & \\
\hline \multicolumn{4}{|l|}{ Model 3} \\
\hline \multicolumn{4}{|l|}{ Group A } \\
\hline \multicolumn{4}{|c|}{ Durable response $\geq 3$ months } \\
\hline Present & 32.4 & $26.0-38.9$ & 0.008 \\
\hline Absent & 15.1 & $13.0-17.3$ & \\
\hline \multicolumn{4}{|c|}{ Durable response $\geq 6$ months } \\
\hline Present & 41.6 & $16.4-66.7$ & 0.079 \\
\hline Absent & 20.1 & $14.9-27.0$ & \\
\hline \multicolumn{4}{|l|}{ Group $A+B$} \\
\hline \multicolumn{4}{|c|}{ Durable response $\geq 3$ months } \\
\hline Present & 36.3 & $24.7-47.9$ & $<0.001$ \\
\hline Absent & 15.2 & $13.6-16.8$ & \\
\hline \multicolumn{4}{|c|}{ Durable response $\geq 6$ months } \\
\hline Present & 50.8 & $28.7-72.9$ & 0.007 \\
\hline Absent & 20.0 & $14.4-25.6$ & \\
\hline
\end{tabular}

CI, confidence interval; HCC, hepatocellular carcinoma; OS, overall survival. 
Fig. 5. Distribution map of tumor burden of the three subclassification models.

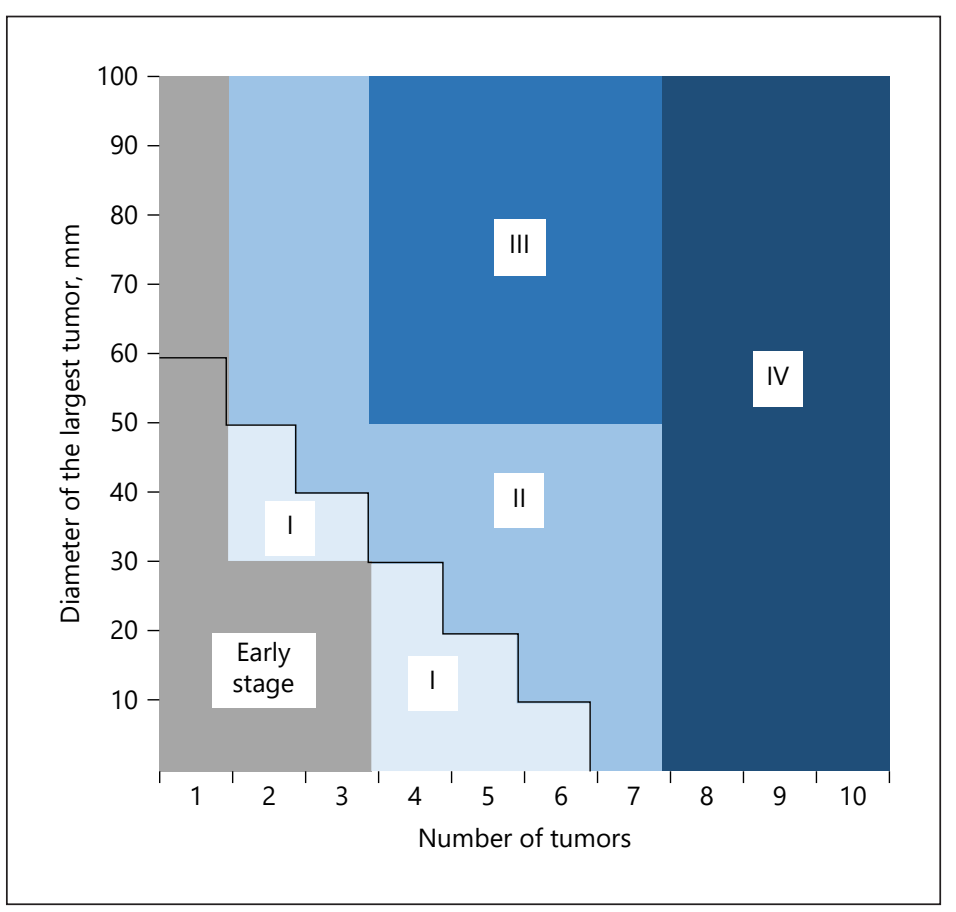

\section{Discussion and Conclusion}

The present study demonstrated that the outcomes of TACE in intermediate-stage HCC patients were strongly correlated with tumor status (number or size). In particular, DRR $\geq 3$ months and $\geq 6$ months in patients with intermediate-stage HCC receiving TACE differed between tumor status, and patients who achieved durable response $\geq 3$ and $\geq 6$ months showed a better prognosis in high-burden intermediate-stage HCC. These results were concordant with previous reports that patients who had achieved durable response after TACE had better prognosis [7]. The point emphasized in the present study was the evaluation of effectiveness in TACE in order to design clinical trials that compare TACE with other promising systemic therapies. In the past, a lot of clinical trials ended in failure due to misunderstanding about the clinical outcomes of standard therapies. We believe that the results of this study may become the standard on effectiveness of TACE for designing clinical trials. Additionally, the present study indicated that previous clinical courses until high-burden intermediate-stage HCC may not be associated with OS. The data may help in increasing the number of study populations when a clinical trial comparing TACE versus systemic therapies would be limited to including eligible patients only to high-burden intermediate-stage HCC.

We defined three subclassification models for the tumor burden of intermediate-stage HCC. Since intermediate-stage HCC includes a heterogeneous population, subclassification of intermediate-stage HCC has become a recent focus. The up-to-seven criteria (subclassification model 1 in the present study) that were created based on the outcomes of liver transplantation [34] had become a probable option for a subclassification model of intermediatestage HCC. The original classification proposed by Bolondi et al. [30] combined tumor factors and liver function assessments according to Child-Pugh score. On the other hand, the present study only focused on tumor burden. Its usefulness as a subclassification model for clinical outcomes of TACE in intermediate-stage HCC patients was subsequently verified in several reports $[30,31,35]$. Thus, we selected the tumor factor of the up-to-seven criteria as a

\section{Karger'}


candidate of subclassification model of intermediate-stage HCC and verified the validity of the up-to-seven criteria for designing future clinical trials. The other two subclassification models were assigned in the present study based on different cutoff values of tumor number and size (subclassification models 2 [five-seven criteria] and 3 [seven lesions criteria]). In the International Union against Cancer manual of clinical oncology (8th edition) [36], the cutoff value of intrahepatic tumor size was $5 \mathrm{~cm}$. Our previous study demonstrated that presence of $>7$ intrahepatic tumors was an independent prognostic factor in patients undergoing TACE [29].

The present study demonstrated that the median OS of patients receiving TACE who were initially diagnosed as high-burden intermediate-stage HCC and those who progressed from low- to high-burden intermediate-stage HCC may be similar in all three subclassification models. Almost all previous studies of TACE reported the median OS from initial TACE and did not consider progression from low- to high-burden intermediate-stage HCC. Our results showed that patients with a previous history of TACE who migrated to high-burden intermediate-stage HCC had the opportunity to enroll in clinical trials, which limited the poor-prognosis population.

We confirmed that both median OS and DRR $\geq 3$ and $\geq 6$ months were different between tumor burdens in the present study. We further investigated patients exceeding the up-toseven criteria, which was a heterogeneous population (Fig. 5), and found that the limited population of those exceeding the up-to-seven criteria (category II in our analysis) showed a better outcome compared with categories III and IV (online suppl. Table 4). Moreover, the median OS from initial TACE was not significantly different between low- and high-burden intermediate-stage HCC patients according to subclassification model 1 (within the up-toseven criteria versus exceeding the up-to-seven criteria). Taken together, the up-to-seven criteria might not be identified as a poor outcome population of TACE, since patients exceeding the up-to-seven criteria included the beneficial population of TACE. Regarding subclassification models 2 and 3, DRR $\geq 3$ and $\geq 6$ months in high-burden patients were similar in both models. In contrast, the OS of high-burden patients according to subclassification model 3 was almost equal but slightly shorter than that of subclassification model 2 .

Based on our results, $<40 \%$ of intermediate-stage HCC patients with Child-Pugh A classified as high-burden disease according to subclassification models 2 and 3 . These rates were from the cohort which excluded patients who had any cancer-related symptom. Although the present study could not correct data of ECOG PS, our cohort seemed to be almost equal to the general population with an indication of TACE. Considering the selection of a subgroup with high-burden intermediate-stage HCC for planning clinical trials which compare systemic therapies with TACE, these limited frequencies in the study population would be up for debate. So far, TACE has worked with matured techniques all over the world. Previous studies and the results of this study have both indicated that TACE could expect durable responses in the beneficial population such as low-burden intermediate-stage HCC [7, 14]. Patients achieving durable response after TACE are mostly free from treatment. In keeping with these points, systemic therapies should be replaced from TACE in a distinct ineffectiveness subpopulation of TACE. We propose that subclassification model 2 is the best option for limiting the number of high-burden patients in future clinical trials. If the expected OS of systemic therapies for the testing arm is not enough long, subclassification model 3 seems to be another option. ICIs may be expected to show a long-lasting response in some populations in various malignancies [20, 21]. In advanced HCC, a single-arm study of nivolumab demonstrated a response rate of $20 \%$, the median duration of response was 9.9 months, and all responders showed an OS $\geq 18$ months [22]. Similarly, a phase II study of pembrolizumab reported a response rate of $17 \%$ and a response duration rate $\geq 9$ months of $77 \%$ [23]. Combined use of tyrosine kinase inhibitors or anti-VEGF monoclonal antibodies with ICI, including atezolizumab combined with bevacizumab, is anticipated as these combinations have the potential

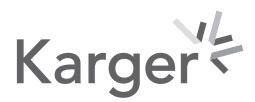




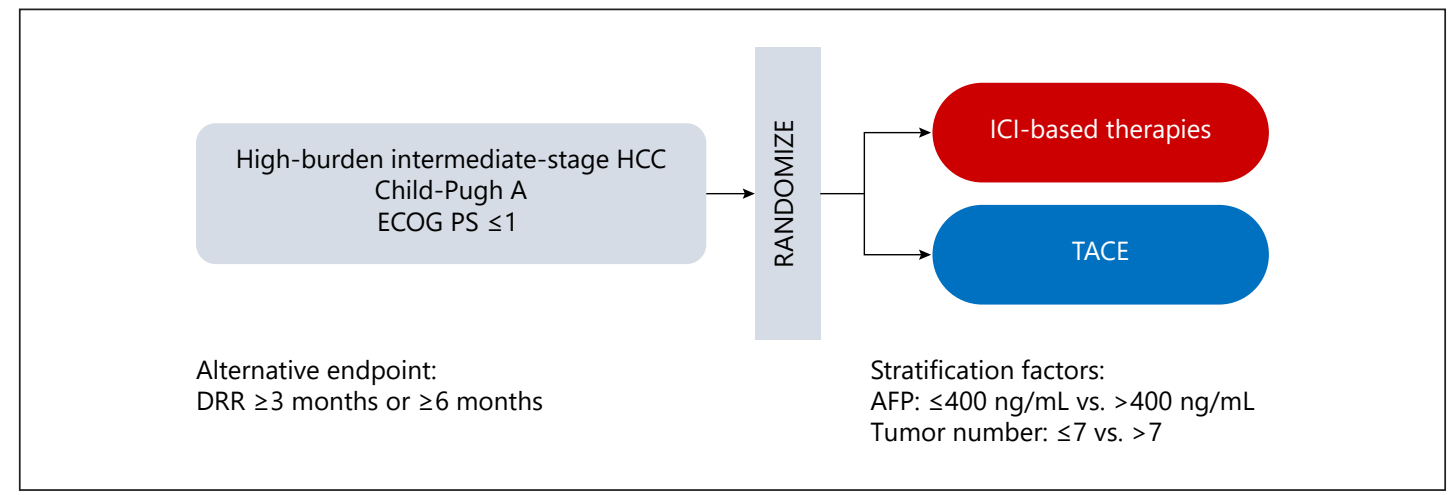

Fig. 6. Example of a clinical trial design utilizing the results from this study. AFP, alpha-fetoprotein; DRR, durable response rate; HCC, hepatocellular carcinoma; ICI, immune checkpoint inhibitor; TACE, transarterial chemoembolization.

to achieve extremely significantly high rates of continuous response in various cancers, including HCC $[25,37,38]$. In particular, the DRR of ICIs or ICI-based therapies could be greater in high-burden intermediate-stage HCC patients receiving TACE, and the latest ICIbased strategies may improve OS in these populations.

TACE aims to achieve continuous tumor control via direct injection of an anticancer agent and embolization of the feeding arteries of liver tumors. Considering this concept, our results showing that durable responses predict OS in patients receiving TACE seemed to be appropriate. OS is currently the gold standard primary endpoint of clinical trials in patients with HCC [39]. Since the median OS of intermediate-stage HCC patients receiving TACE is around 30 months $[8,40]$ and the variation of post-TACE therapy is assumed, it is not easy to design clinical trials for intermediate-stage HCC that set the primary endpoint as OS and TACE for the standard arm. Therefore, we believe that DRR $\geq 3$ or $\geq 6$ months is a possible candidate as an alternative endpoint for clinical trials comparing TACE and ICI-based therapies, particularly randomized phase II studies (Fig. 6).

When designing clinical trials that compare TACE and ICI-based therapies, several issues need to be addressed. First, external validation of the results of the present study is strongly required through other cohorts of intermediate-stage HCC patients who received TACE, as this study was done on the database of a single-center experience. Furthermore, DRR $\geq 3$ months or $\geq 6$ months as surrogate endpoints of survival require validation in HCC patients who receive ICIs. With regard to this point, we strongly desire to conduct a validation analysis by pooling the data of published clinical trials of ICIs in HCC patients [22, 23, 25, 37, 38]. Additionally, the most suitable stratification factors for randomization need to be discussed. Several scoring systems for predicting the survival outcomes of TACE have been developed during the previous decade, and these parameters appear to provide helpful information [41-44]. Most scoring systems of TACE consisted of both tumor factors and parameters of liver function. Kadalayil et al. [41] advocated the Hepatoma Arterial Embolization Prognostic score that includes AFP for calculation scoring along with albumin, bilirubin, and tumor size. Regarding the stratification factors of systemic therapies, tumor factors and AFP value have been commonly used, and liver function has been selected only when subjects with worse liver function were included in trials. In sum, we consider that tumor factors and AFP value are the most appropriate stratification factors available (Fig. 6). Since the selection of stratification factors has strongly affected the outcomes of randomized clinical trials mainly in HCC, we should continue discussing this point in the future $[15,45,46]$.

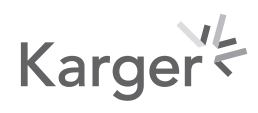


In conclusion, the present study demonstrates the outcomes of TACE in patients with intermediate-stage HCC with heterogeneous tumor status and progression processes during their clinical courses. Our results suggest that high-burden intermediate-stage HCC, especially in the limited population of beyond the up-to-seven criteria, is associated with a lower chance of achieving DDR $\geq 3$ months or $\geq 6$ months and poor prognosis after receiving TACE. High-burden intermediate-stage HCC patients with confirmed DDR $\geq 3$ months or $\geq 6$ months showed better survival outcomes. Our results provide useful information for designing clinical trials comparing TACE and ICIs and demonstrate the basis for selecting a patient population that would not benefit from TACE and setting DRR $\geq 3$ or $\geq 6$ months as an alternative endpoint.

\section{Acknowledgement}

The authors would like to thank Yohei Kawasaki and Katsuyuki Haino for advice on statistical analysis. They would also like to thank Enago (www.enago.jp) for English language review.

\section{Statement of Ethics}

All procedures were in accordance with the ethical standards of the institutional and/or national research committee and with the 1964 Helsinki Declaration and its later amendments or comparable ethical standards. The Chiba University Research Ethics Committee approved this study (clinical trial No. 2247). For this type of study, formal consent was not required.

\section{Conflict of Interest Statement}

S. Ogasawara received honoraria from Bayer, Eisai, and Eli Lilly, consulting or advisory fees from Bayer, Eisai, Merck \& Co., Inc., Chugai Pharma, Eli Lilly, and AstraZeneca, and research grants from Bayer and Eisai. N. Kato received honoraria from Bayer, Eisai, Sumitomo Dainippon Pharma, and Merck \& Co., Inc., consulting or advisory fees from Bayer and Eisai, and research grants from Bayer and Eisai. The other authors involved in this study have nothing to declare regarding funding or conflicts of interest with respect to this study.

\section{Funding Sources}

This research received no external funding.

\section{Author Contributions}

All authors approved the final version of the manuscript. K. Koroki: acquisition of data, analysis and interpretation of data, drafting of the manuscript. S. Ogasawara: acquisition of data, analysis and interpretation of data, study design, critical revision of the manuscript. Y. Ooka: acquisition of data, study supervision. K. Kobayashi: acquisition of data, analysis and interpretation of data. H. Kanzaki, K. Kanayama, S. Maruta, T. Maeda, M. Yokoyama, T. Wakamatsu, M. Inoue, S. Kiyono, M. Nakamura, N. Kanogawa, T. Saito, T. Kondo, E. Suzuki, S. Nakamoto, S. Yasui, A. Tawada, T. Chiba, M. Arai, T. Kanda, H. Maruyama: acquisition of data. J. Kato, S. Kuboki, M. Ohtsuka, M. Miyazaki, O. Yokosuka, N. Kato: study supervision. 


\begin{tabular}{|c|c|}
\hline Liver Cancer 2020;9:596-6 & \\
\hline DOI: 10.1159/000508809 & $\begin{array}{l}\text { (c) } 2020 \text { The Author(s). Published by S. Karger AG, Basel } \\
\text { www.karger.com/lic }\end{array}$ \\
\hline
\end{tabular}

Koroki et al.: Designing Clinical Trials for Intermediate-Stage HCC

\section{References}

1 Forner A, Reig M, Bruix J. Hepatocellular carcinoma. Lancet. 2018 Mar;391(10127):1301-14.

2 Park JW, Chen M, Colombo M, Roberts LR, Schwartz M, Chen PJ, et al. Global patterns of hepatocellular carcinoma management from diagnosis to death: the BRIDGE Study. Liver Int. 2015 Sep;35(9):2155-66.

3 Llovet JM, Bruix J. Systematic review of randomized trials for unresectable hepatocellular carcinoma: chemoembolization improves survival. Hepatology. 2003 Feb;37(2):429-42.

4 Galle PR, Forner A, Llovet JM, Mazzaferro V, Piscaglia F, Raoul JL, et al.; European Association for the Study of the Liver. EASL Clinical Practice Guidelines: management of hepatocellular carcinoma. J Hepatol. 2018 Jul; 69(1):182-236.

5 Omata M, Cheng AL, Kokudo N, Kudo M, Lee JM, Jia J, et al. Asia-Pacific clinical practice guidelines on the management of hepatocellular carcinoma: a 2017 update. Hepatol Int. 2017 Jul;11(4):317-70.

6 Lencioni R, Llovet JM, Han G, Tak WY, Yang J, Guglielmi A, et al. Sorafenib or placebo plus TACE with doxorubicin-eluting beads for intermediate stage HCC: the SPACE trial. J Hepatol. 2016 May;64(5):1090-8.

7 Llovet JM, Real MI, Montaña X, Planas R, Coll S, Aponte J, et al.; Barcelona Liver Cancer Group. Arterial embolisation or chemoembolisation versus symptomatic treatment in patients with unresectable hepatocellular carcinoma: a randomised controlled trial. Lancet. 2002 May;359(9319):1734-9.

8 Kudo M, Cheng AL, Park JW, Park JH, Liang PC, Hidaka H, et al. Orantinib versus placebo combined with transcatheter arterial chemoembolisation in patients with unresectable hepatocellular carcinoma (ORIENTAL): a randomised, double-blind, placebo-controlled, multicentre, phase 3 study. Lancet Gastroenterol Hepatol. 2018 Jan;3(1):37-46.

9 Llovet JM, Ricci S, Mazzaferro V, Hilgard P, Gane E, Blanc JF, et al.; SHARP Investigators Study Group. Sorafenib in advanced hepatocellular carcinoma. N Engl J Med. 2008 Jul;359(4):378-90.

10 Cheng AL, Kang YK, Chen Z, Tsao CJ, Qin S, Kim JS, et al. Efficacy and safety of sorafenib in patients in the AsiaPacific region with advanced hepatocellular carcinoma: a phase III randomised, double-blind, placebocontrolled trial. Lancet Oncol. 2009 Jan;10(1):25-34.

11 Kokudo N, Hasegawa K, Akahane M, Igaki H, Izumi N, Ichida T, et al. Evidence-based clinical practice guidelines for hepatocellular carcinoma: The Japan Society of Hepatology 2013 update (3rd JSH-HCC Guidelines). Hepatol Res. 2015 Jan;45(2):123-7.

12 Ogasawara S, Chiba T, Ooka Y, Kanogawa N, Motoyama T, Suzuki E, et al. Efficacy of sorafenib in intermediatestage hepatocellular carcinoma patients refractory to transarterial chemoembolization. Oncology. 2014; 87(6):330-41.

13 Arizumi T, Ueshima K, Minami T, Kono M, Chishina H, Takita M, et al. Effectiveness of sorafenib in patients with transcatheter arterial chemoembolization (TACE) refractory and intermediate-stage hepatocellular carcinoma. Liver Cancer. 2015 Dec;4(4):253-62.

14 Radosavljevic MP, Kudo M, Raoul JL, Lee HC, Decaens T, Heo J, et al. Outcomes of patients with hepatocellular carcinoma (HCC) treated with transarterial chemoembolization (TACE): global OPTIMIS final analysis. J Clin Oncol. 2018;36(Suppl):abstract 4018.

15 Bruix J, Qin S, Merle P, Granito A, Huang YH, Bodoky G, et al.; RESORCE Investigators. Regorafenib for patients with hepatocellular carcinoma who progressed on sorafenib treatment (RESORCE): a randomised, doubleblind, placebo-controlled, phase 3 trial. Lancet. 2017 Jan;389(10064):56-66.

16 Kudo M, Finn RS, Qin S, Han KH, Ikeda K, Piscaglia F, et al. Lenvatinib versus sorafenib in first-line treatment of patients with unresectable hepatocellular carcinoma: a randomised phase 3 non-inferiority trial. Lancet. 2018 Mar;391(10126):1163-73.

17 Abou-Alfa GK, Meyer T, Cheng AL, El-Khoueiry AB, Rimassa L, Ryoo BY, et al. Cabozantinib in patients with advanced and progressing hepatocellular carcinoma. N Engl J Med. 2018 Jul;379(1):54-63.

18 Zhu AX, Kang YK, Yen CJ, Finn RS, Galle PR, Llovet JM, et al.; REACH-2 study investigators. Ramucirumab after sorafenib in patients with advanced hepatocellular carcinoma and increased $\alpha$-fetoprotein concentrations (REACH-2): a randomised, double-blind, placebo-controlled, phase 3 trial. Lancet Oncol. 2019 Feb;20(2):28296.

19 Ogasawara S, Ooka Y, Koroki K, Maruta S, Kanzaki H, Kanayama K, et al. Switching to systemic therapy after locoregional treatment failure: definition and best timing. Clin Mol Hepatol. 2020 Apr;26(2):155-62.

20 Topalian SL, Sznol M, McDermott DF, Kluger HM, Carvajal RD, Sharfman WH, et al. Survival, durable tumor remission, and long-term safety in patients with advanced melanoma receiving nivolumab. J Clin Oncol. 2014 Apr;32(10):1020-30.

21 Gettinger SN, Horn L, Gandhi L, Spigel DR, Antonia SJ, Rizvi NA, et al. Overall survival and long-term safety of nivolumab (Anti-Programmed Death 1 Antibody, BMS-936558, ONO-4538) in patients with previously treated advanced non-small-cell lung cancer. J Clin Oncol. 2015 Jun;33(18):2004-12.

22 El-Khoueiry AB, Sangro B, Yau T, Crocenzi TS, Kudo M, Hsu C, et al. Nivolumab in patients with advanced hepatocellular carcinoma (CheckMate 040): an open-label, non-comparative, phase 1/2 dose escalation and expansion trial. Lancet. 2017 Jun;389(10088):2492-502.

23 Zhu AX, Finn RS, Edeline J, Cattan S, Ogasawara S, Palmer D, et al.; KEYNOTE-224 investigators. Pembrolizumab in patients with advanced hepatocellular carcinoma previously treated with sorafenib (KEYNOTE224): a non-randomised, open-label phase 2 trial. Lancet Oncol. 2018 Jul;19(7):940-52. 
24 Kudo M. Targeted and immune therapies for hepatocellular carcinoma: predictions for 2019 and beyond. World J Gastroenterol. 2019 Feb;25(7):789-807.

25 Cheng AL, Qin S, Ikeda M, Galle P, Ducreux M, Zhu A, et al. IMbrave150: efficacy and safety results from a ph III study evaluating atezolizumab (atezo) + bevacizumab (bev) vs sorafenib (Sor) as first treatment (tx) for patients (pts) with unresectable hepatocellular carcinoma (HCC). Ann Oncol. 2019;30(Suppl 9):186-7.

26 Piscaglia F, Ogasawara S. Patient Selection for Transarterial Chemoembolization in Hepatocellular Carcinoma: Importance of Benefit/Risk Assessment. Liver Cancer. 2018 Mar;7(1):104-19.

27 Kudo M. A New Treatment Option for Intermediate-Stage Hepatocellular Carcinoma with High Tumor Burden: Initial Lenvatinib Therapy with Subsequent Selective TACE. Liver Cancer. 2019 Oct;8(5):299-311.

28 Cescon M, Vetrone G, Grazi GL, Ramacciato G, Ercolani G, Ravaioli M, et al. Trends in perioperative outcome after hepatic resection: analysis of 1500 consecutive unselected cases over 20 years. Ann Surg. 2009 Jun; 249(6):995-1002.

29 Ogasawara S, Chiba T, Ooka Y, Kanogawa N, Motoyama T, Suzuki E, et al. A prognostic score for patients with intermediate-stage hepatocellular carcinoma treated with transarterial chemoembolization. PLoS One. 2015 Apr;10(4):e0125244.

30 Bolondi L, Burroughs A, Dufour JF, Galle PR, Mazzaferro V, Piscaglia F, et al. Heterogeneity of patients with intermediate (BCLC B) Hepatocellular Carcinoma: proposal for a subclassification to facilitate treatment decisions. Semin Liver Dis. 2012 Nov;32(4):348-59.

31 Kudo M, Arizumi T, Ueshima K, Sakurai T, Kitano M, Nishida N. Subclassification of BCLC B Stage Hepatocellular Carcinoma and Treatment Strategies: Proposal of Modified Bolondi's Subclassification (Kinki Criteria). Dig Dis. 2015 Oct;33(6):751-8.

32 Lencioni R, Llovet JM. Modified RECIST (mRECIST) assessment for hepatocellular carcinoma. Semin Liver Dis. 2010 Feb;30(1):52-60.

33 Andtbacka RH, Kaufman HL, Collichio F, Amatruda T, Senzer N, Chesney J, et al. Talimogene Laherparepvec Improves Durable Response Rate in Patients With Advanced Melanoma. J Clin Oncol. 2015 Sep;33(25): 2780-8.

34 Mazzaferro V, Llovet JM, Miceli R, Bhoori S, Schiavo M, Mariani L, et al.; Metroticket Investigator Study Group. Predicting survival after liver transplantation in patients with hepatocellular carcinoma beyond the Milan criteria: a retrospective, exploratory analysis. Lancet Oncol. 2009 Jan;10(1):35-43.

35 Yasui Y, Tsuchiya K, Kurosaki M, Takeguchi T, Takeguchi Y, Okada M, et al. Up-to-seven criteria as a useful predictor for tumor downstaging to within Milan criteria and Child-Pugh grade deterioration after initial conventional transarterial chemoembolization. Hepatol Res. 2018 May;48(6):442-50.

36 Kamarajah SK, Frankel TL, Sonnenday C, Cho CS, Nathan H. Critical evaluation of the American Joint Commission on Cancer (AJCC) 8th edition staging system for patients with Hepatocellular Carcinoma (HCC): a Surveillance, Epidemiology, End Results (SEER) analysis. J Surg Oncol. 2018 Mar;117(4):644-50.

37 Stein S, Pishvaian MJ, Lee SM, Lee KH, Hernandez S, Kwan A, et al. Safety and clinical activity of 1L atezolizumab + bevacizumab in a phase Ib study in hepatocellular carcinoma (HCC). J Clin Oncol.2018;36(Suppl):abstract 4074.

38 Ikeda M, Sung MW, Kudo M, et al. A phase $1 \mathrm{~b}$ trial of lenvatinib (LEN) plus pembrolizumab (PEM) in patients (pts) with unresectable hepatocellular carcinoma (uHCC). J Clin Oncol. 2018;36(Suppl):abstract 4076.

39 Llovet JM, Montal R, Villanueva A. Randomized trials and endpoints in advanced HCC: role of PFS as a surrogate of survival. J Hepatol. 2019 Jun; 70(6):1262-77.

40 Kudo M, Han G, Finn RS, Poon RT, Blanc JF, Yan L, et al. Brivanib as adjuvant therapy to transarterial chemoembolization in patients with hepatocellular carcinoma: A randomized phase III trial. Hepatology. 2014 Nov; 60(5):1697-707.

41 Kadalayil L, Benini R, Pallan L, O’Beirne J, Marelli L, Yu D, et al. A simple prognostic scoring system for patients receiving transarterial embolisation for hepatocellular cancer. Ann Oncol. 2013 Oct;24(10):2565-70.

42 Sieghart W, Hucke F, Pinter M, Graziadei I, Vogel W, Müller C, et al. The ART of decision making: retreatment with transarterial chemoembolization in patients with hepatocellular carcinoma. Hepatology. 2013 Jun;57(6): 2261-73.

43 Hucke F, Pinter M, Graziadei I, Bota S, Vogel W, Müller C, et al. How to STATE suitability and START transarterial chemoembolization in patients with intermediate stage hepatocellular carcinoma. J Hepatol. 2014 Dec; 61(6):1287-96.

44 Pinato DJ, Arizumi T, Jang JW, Allara E, Suppiah PI, Smirne C, et al. Combined sequential use of HAP and ART scores to predict survival outcome and treatment failure following chemoembolization in hepatocellular carcinoma: a multi-center comparative study. Oncotarget. 2016 Jul;7(28):44705-18.

45 Llovet JM, Decaens T, Raoul JL, Boucher E, Kudo M, Chang C, et al. Brivanib in patients with advanced hepatocellular carcinoma who were intolerant to sorafenib or for whom sorafenib failed: results from the randomized phase III BRISK-PS study. J Clin Oncol. 2013 Oct;31(28):3509-16.

46 Llovet JM, Hernandez-Gea V. Hepatocellular carcinoma: reasons for phase III failure and novel perspectives on trial design. Clin Cancer Res. 2014 Apr;20(8):2072-9. 